\title{
In-plane anisotropy of coercive field in permalloy square ring arrays
}

\author{
A. V. Goncharov and A. A. Zhukov ${ }^{\text {a) }}$ \\ School of Physics and Astronomy, University of Southampton, Southampton SO17 1BJ, United Kingdom \\ V. V. Metlushko \\ Department of Electrical and Computer Engineering, University of Illinois at Chicago, \\ Chicago, Illinois 60607-0024 \\ G. Bordignon and H. Fangohr \\ School of Engineering Sciences, University of Southampton, Southampton SO17 1BJ, United Kingdom \\ G. Karapetrov \\ Materials Science Division, Argonne National Laboratory, 9700 South Cass Avenue, \\ Argonne, Illinois 60439 \\ B. Ilic \\ Cornell Nanofabrication Facility and School of Applied and Engineering Physics, Cornell University, \\ Ithaca, New York 14853 \\ P. A. J. de Groot \\ School of Physics and Astronomy, University of Southampton, Southampton, SO17 1BJ, United Kingdom
}

(Presented on 2 November 2005; published online 24 April 2006)

Magnetic ring arrays are promising candidates for application in magnetic random access memory devices. The magnetic reversal processes and anisotropy of the coercivity in arrays of square-shaped nanorings with different spacings were investigated by vector magneto-optical Kerr effect magnetometry, magnetic force microscopy, and micromagnetic simulations. Two-step magnetization reversal demonstrates fourfold symmetry in the film plane resulting from the shape anisotropy in rings. Our numerical simulations show good agreement with the experiment. (C) 2006 American Institute of Physics. [DOI: 10.1063/1.2171949]

\section{INTRODUCTION}

Magnetic nanostructures demonstrate a wealth of magnetic properties, which are extremely sensitive to shapes and the order of nanoelements strongly interacting via exchange and dipolar coupling. ${ }^{1}$ From a technological point of view, such materials are important for applications in magnetic recording and, in particular, for rapidly developing magnetic random access memory (MRAM) devices. ${ }^{2}$ Magnetic nanorings are among the most promising candidates for the future MRAM applications. ${ }^{3}$ Their main advantage stems from the presence of a hole in the center of the ring, which eliminates the highly energetic vortex core existing in a dot element. Recently, magnetic properties of arrays with square-shaped nanorings have been reported in several publications. ${ }^{4-6}$ However, these studies have concentrated on magnetic reversal for magnetic fields along the main symmetry directions and specific arrays with well-separated ring elements. In this work we explore the in-plane anisotropy of coercive field in permalloy square nanoring arrays with different spacings to understand the impact of shape-induced magnetic anisotropy and interelement dipolar interactions.

\section{SAMPLE PREPARATION AND MAGNETIC MEASUREMENTS}

In this work we study three arrays of permalloy nanorings with different interelement spacing distances of 45,70 , and $200 \mathrm{~nm}$. Below we refer to these samples as P45, P70,

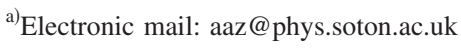

and P200, respectively. Figure 1 shows a scanning electron microscopy (SEM) image for the first array. In all arrays the dimensions of square rings are $950 \mathrm{~nm}$ for the lateral size, $200 \mathrm{~nm}$ for the side width, and $25 \mathrm{~nm}$ for the thickness. In the preparation a standard 3 in. (100) silicon wafer was spin coated with bilayer resists polymethyl methacrylate (PMMA) and P(MMA-MAA) copolymers and baked. The wafer was then placed into a Raith e-beam writer and desired patterns consisting of ring elements were written. Using e-beam evaporation in a high-vacuum chamber with a typical evaporation rate of $0.1-0.2 \AA / \mathrm{s}$ a $25 \mathrm{~nm}$ permalloy film was deposited onto the holes in the resist. After a lift-off in ac-

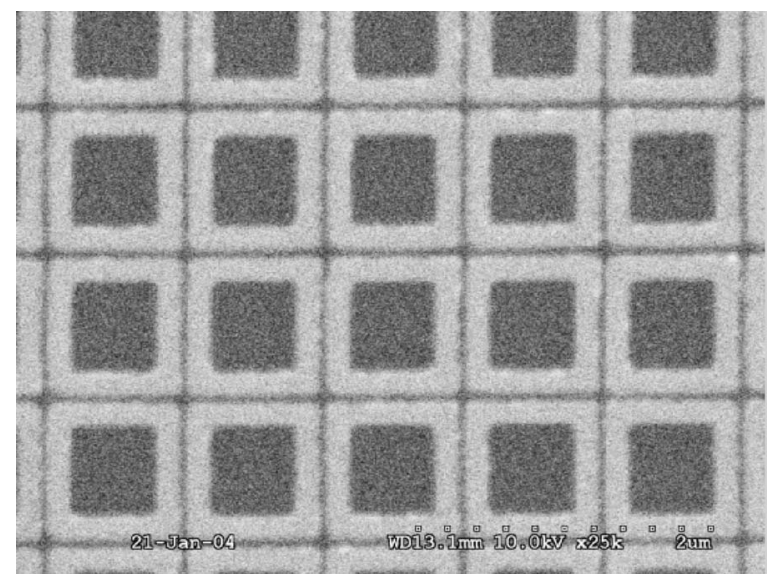

FIG. 1. SEM image of the P45 array of square-shaped $950 \mathrm{~nm}$ nanorings with $45 \mathrm{~nm}$ separation. 


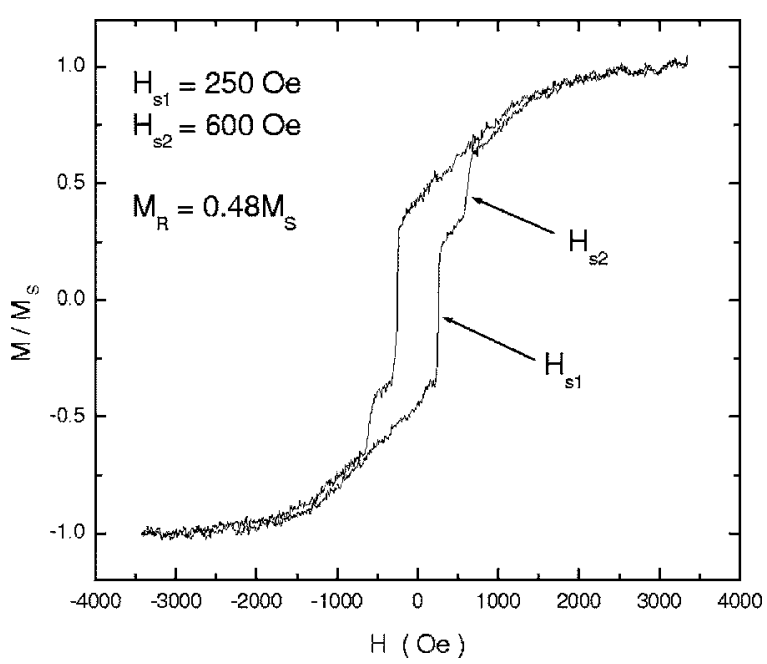

FIG. 2. Magnetization loops measured by longitudinal MOKE showing the component of the magnetization parallel to the magnetic field in the sample $\mathrm{P} 70$. The direction of the field is $10^{\circ}$ away from the edge of the ring.

etone the arrays of magnetic permalloy rings were obtained. Using longitudinal magneto-optical Kerr effect (MOKE) with both longitudinal and transverse magnetic fields we have measured the hysteresis loops for parallel and transverse components of magnetization. ${ }^{7}$ Measurements of $M$ $-H$ curves were performed at room temperature for magnetic fields in the film plane with various angles between the field direction and the edge of the rings. The magnetic force microscope (MFM) images were obtained with a Digital Instruments 3000 scanning probe microscope using lift mode with a fly height of $100 \mathrm{~nm}$ and a standard low-moment ferromagnetic tip. Magnetic simulations have been done using OOMMF software suite. ${ }^{8}$

\section{RESULTS AND DISCUSSION}

Our measurements demonstrate that magnetization reversal is normally realized by a two-step process and is affected strongly by the direction of the applied magnetic field.

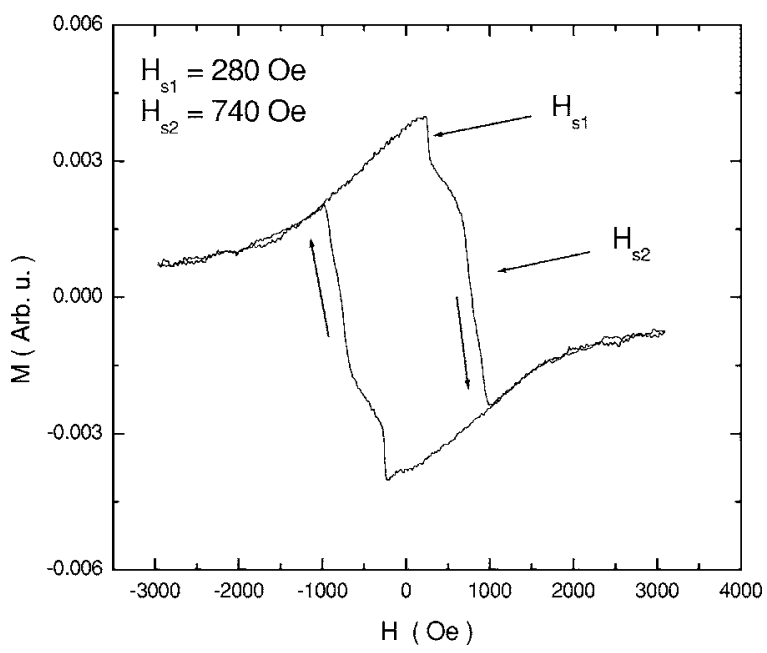

FIG. 3. Magnetization loops measured by a vector-MOKE technique showing components of the magnetization transverse to the magnetic field in the sample P200. The direction of the field is $10^{\circ}$ away from the edge of the ring.

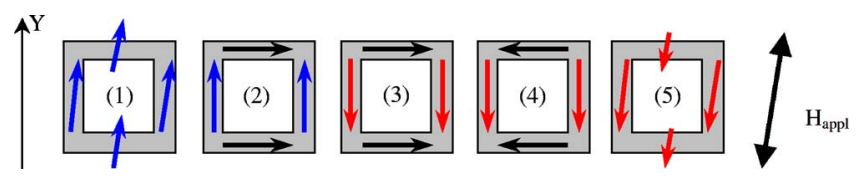

FIG. 4. (Color online) A schematic representation of results from micromagnetic calculations with the magnetic field applied along the edge of the ring with $10^{\circ}$ offset clockwise from the $Y$ direction.

Figure 2 shows a MOKE hysteresis loop for the parallel component of magnetization when the magnetic field is applied at an angle of $\varphi=10^{\circ}$ from the edge of the ring. The $M-H$ curve has two characteristic steps corresponding to switching fields: $H_{s 1}=250 \mathrm{Oe}$ and $H_{s 2}=500 \mathrm{Oe}$. The first step crosses $M / M_{s}=0$ and corresponds to the standard definition of coercivity. This type of behavior reveals that during the reversal process the magnetization switches twice.

Similar two-step switching is also demonstrated by the transverse component of the magnetic moment as can be seen from Fig. 3. However, in this case the second magnetization step is bigger and would in this case define the standard coercive field. Comparing these two $M$ components the underlying magnetic transformation can be understood from a simple qualitative analysis ${ }^{4-6}$ based on independent switching for different sides of the square ring. Such an approach finds confirmation in our numerical simulations. From the comparison of both magnetization components we may conclude that a gradual rotation of magnetization from the field direction towards alignment with the borders of the transverse sides of the ring is realized before the first step. This corresponds to a transition from a saturated field-aligned state (1) to a so called onion state (2) in Fig. 4. At the first switching the parallel magnetic moment component is mostly affected, which indicates the magnetization reversal for ring sides parallel to the field. Dominant change in the transverse magnetization for the second switching means that the second step corresponds to a reversal of magnetic moments in the transverse sides. Therefore, in Fig. 4 the first step gives us a $90^{\circ}$ rotation of the onion state from (2) to (3). The second step reverses the onion state and corresponds to a transition from (3) to (4). At high fields the saturated state (5) is reached.

Our numerical simulations using typical parameters for permalloy $A=13 \mathrm{pJ} / \mathrm{m}, \mu_{0} M_{s}=1 \mathrm{~T}, K=0$, and a cell size of $5 \mathrm{~nm}$ closely reproduce the experimental results and the transformations suggested by our qualitative analysis. The parallel component of magnetization calculated for $\varphi=10^{\circ}$ is shown in Fig. 5. Existence of the onion configuration (shown by white arrows) for the remanent state is also confirmed by MFM imaging (Fig. 6), which in addition demonstrates strong interaction between the onion states in neighboring squares. Similarly to well-separated rings ${ }^{4,5}$ dark and white spots are observed along the $135^{\circ}$ diagonal at head-to-head and tail-to-tail points. A reduced contrast of this image is probably due to the smaller element spacing in our array, which suppresses the stray field.

In Fig. 7 we present the dependence of both switching fields on the angle $\varphi$ between the applied field and the edge of the sample. Pronounced fourfold symmetry is apparent. Assuming that the reversal mechanism is dominated by the 


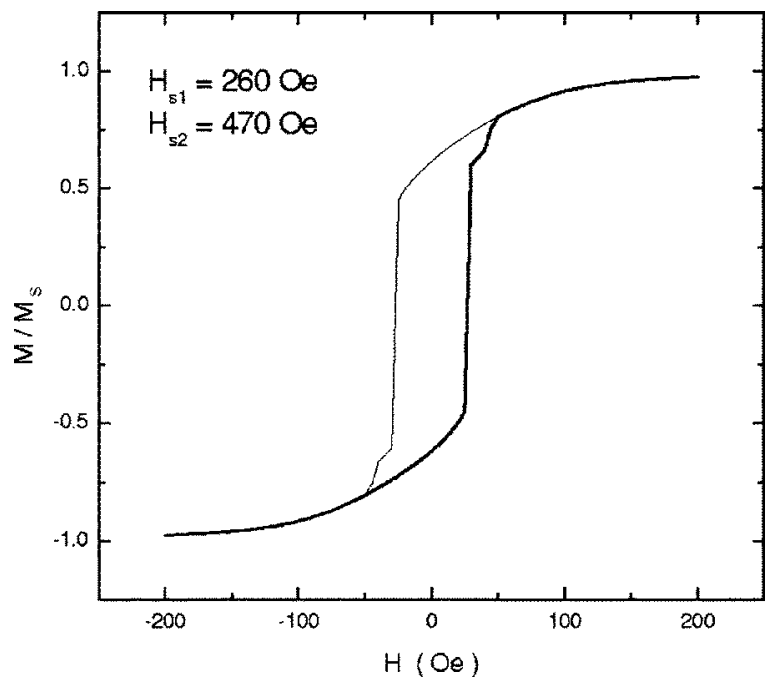

FIG. 5. $M-H$ loop calculated using OoMMF code, showing result for $\mathbf{M}_{\|}$ component of the magnetization.

magnetostatic energy $E_{\mathrm{ms}}$ we can reproduce our experimental behavior in the framework of a simple model based on Stoner-Wohlfarth approach. ${ }^{9}$ Describing the energy variations as $\Delta N M_{s}^{2} \sin ^{2} \varphi / 2$ and $\Delta N M_{s}^{2} \cos ^{2} \varphi / 2$ for the parallel and transverse sides of the ring, respectively, and taking $\Delta H_{c}=\Delta E_{\mathrm{ms}} / M_{s}$ we obtain the best fit for $\Delta N=0.08$, which is well comparable with the difference in the demagnetizing factors for ring sides of $\Delta N=0.11$.

With the magnetic field parallel to the diagonal direction $\left(\varphi=45^{\circ}\right)$ we would expect a single transition from the symmetry consideration, which also agrees with the numerical simulations. Presence of two transitions may originate from small misalignment in the sample plane or some mosaic spread in the effective directions of squares resulting from the accuracy of lithography. The possible impact of interelement dipolar interaction is planned to be explored by numerical simulations.

Experimental results for the first switching field $H_{s 1}$

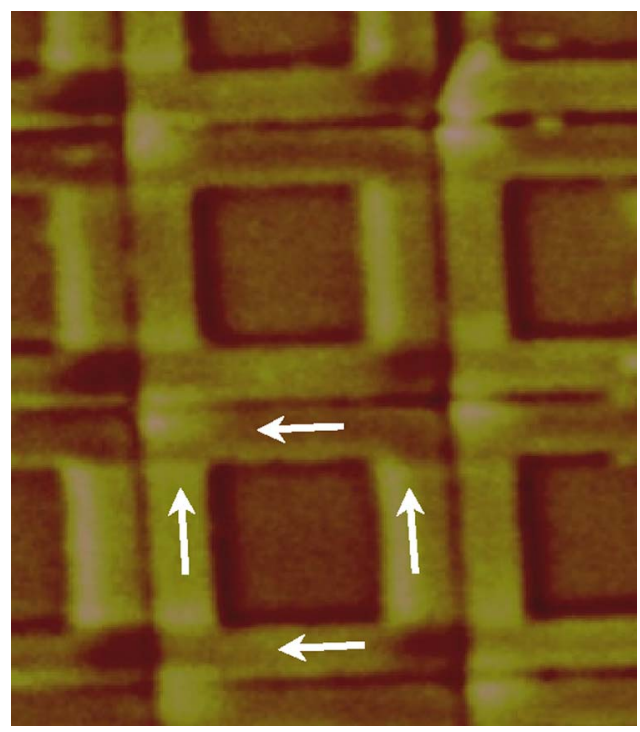

FIG. 6. (Color online) MFM image of P45 array in the remanent state. The arrows show the direction of magnetization for the onion state.

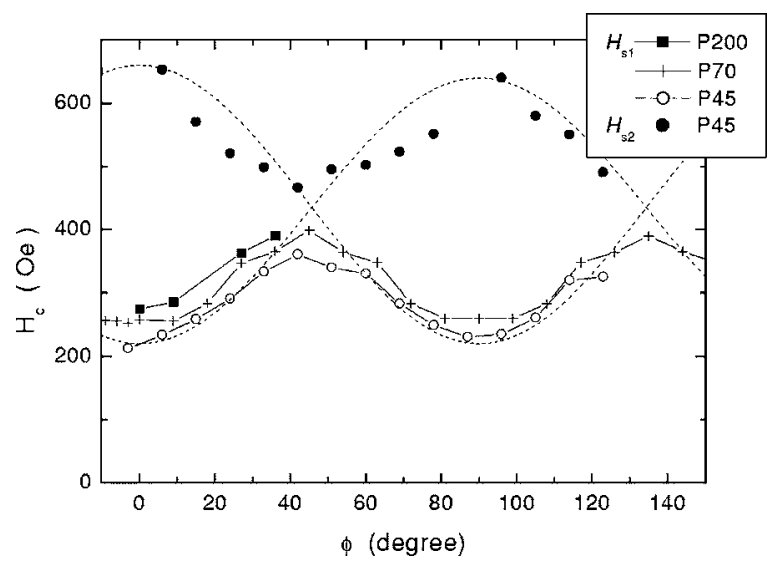

FIG. 7. Angular dependences of the two switching fields for the studied samples. The dashed line shows illustrative angular behavior for simplified magnetization switching governed by magnetostatic energy of ring sides.

demonstrate the relatively small effect of spacing between the rings. As the spacing decreases, the coercivity slowly decreases. This can be explaned by the increased coupling between the square rings, which decreases demagnetizing effects and make the behavior more similar to an unpatterned film. Indeed the demagnetizing field of noncompensated poles on the surface of a ring tend to be compensated by the opposite poles of neighboring element decreasing the shape anisotropy and resulting into decreased coercivity for closerspaced elements.

\section{CONCLUSIONS}

In conclusion we have studied the in-plane anisotropy of the magnetic properties in two-dimensional arrays of square nanorings. The pronounced two-step magnetization processes have been analyzed using vector MOKE. The shape of the rings affects the magnetization process significantly leading to a fourfold anisotropy of the switching fields. The experimental results demonstrate a rather weak impact of interelement interactions with decreasing coercivity for smaller interelement spacing.

\section{ACKNOWLEDGMENTS}

This work was supported by the U.K. Engineering and Physical Sciences Research Council (Grants Nos. GR/ S13200/01 and EP/C511786/1) and by the U.S. National Science Foundation (Grant No. DMR-0210519).

${ }^{1}$ R. Skomski, J. Phys.: Condens. Matter 15, R841 (2003).

${ }^{2}$ A. Moser, K. Takano, D. T. Margulies, M. Albrecht, Y. Sonobe, Y. Ikeda, S. Sun, and E. E. Fullerton, J. Phys. D 35 R157 (2002).

${ }^{3}$ J. G. Zhu, Y. Zheng, and G. A. Prinz, J. Appl. Phys. 87, 6668 (2000).

${ }^{4}$ X. Zhu, P. Grutter, V. Metlushko, and B. Ilic, J. Appl. Phys. 93, 7059 (2003).

${ }^{5}$ P. Vavassori, M. Grimsditch, V. Novosad, V. Metlushko, and B. Ilic, Phys. Rev. B 67, 134429 (2003).

${ }^{6}$ P. Vavassori, M. Grimsditch, V. Novosad, V. Metlushko, and B. Ilic, J. Appl. Phys. 93, 7900 (2003).

${ }^{7}$ Z. J. Yang and M. R. Scheifein, J. Appl. Phys. 74, 6810 (1993).

${ }^{8}$ M. J. Donahue and D. G. Porter, OOMMF Version 1.0; http://math.nist.gov/ oommf

${ }^{9}$ E. C. Stoner and E. P. Wolfarth, Philos. Trans. R. Soc. London, Ser. A 240, 599 (1948) 